\title{
2010, les 50 ans du laser en France
}

Le $X^{\mathrm{e}}$ siècle a été celui de l'électronique et de la microélectronique, le $\mathrm{XXI}^{\mathrm{e}}$ siècle sera celui de la photonique et du laser,

1- Comité National d'Optique et Photonique

2- Association Française des industries de l'Optique et de la Photonique
Fruit d'un travail de toutes les forces vives qui gravitent autour du laser, I'anniversaire de ses 50 ans a illustré le consensus qui prévaut en France pour sa promotion et ses applications.

Il y a 50 ans, le 16 mai 1960, naissait le premier laser, le laser à rubis découvert par Theodore Maiman aux États-Unis. Cette nouvelle technique de création d'une lumière ordonnée, pure et concentrée, directionnelle, très intense, monochromatique, s'est installée depuis dans notre quotidien, de l'industrie à la recherche fondamentale et appliquée, des télécommunications optiques à la médecine, de l'environnement à la biophotonique, du stockage à la lecture et au transport de données, de la simulation des étoiles à la mesure des distances...

La découverte du laser a élargi le champ du savoir. Elle a eu rapidement des conséquences sur la vie quotidienne et l'ensemble de la vie économique : des graveurs/lecteurs DVD à nos imprimantes ou aux lecteurs de codes à barres, de la découpe/soudage/perçage au marquage industriel, de la transmission de l'information par fibres optiques via Internet à la fabrication des microprocesseurs, de l'utilisation des lasers pour la défense aux mesures de distances Terre-lune ou Terre-satellites, des diagnostics médicaux in vivo à

\section{MANIFESTATIONS NATIONALES}

- Journée de lancement des 50 ans du laser en France, 7 janvier, Palais de la découverte. Sous le patronage de Claudie Haigneré, Présidente du Palais de la Découverte et de la Cité des Sciences et de I'Industrie, et avec la participation exceptionnelle de Claude Cohen-Tannoudji, prix Nobel de Physique.

- 50 ans du laser dans la Ville Lumière, 22 juin, Auditorium du Louvre. Conférences de prestige international, grâce à Gérard Mourou, avec la participation exceptionnelle de Charles Townes, I'inventeur du laser, et de cinq autres prix Nobel, ainsi que du gotha du laser mondial. Ce fut une des conférences internationales les plus grandioses des 50 dernières années dans le monde du laser.

- Journée de rencontres avec les jeunes et le grand public, 23 juin, École polytechnique. Sessions pédagogiques et table ronde-rencontre entre les jeunes et six prix Nobel, événement exceptionnel avec la participation de plus de 800 jeunes, lycéens et étudiants, venus de toute la France.

- Salon PRI/OPTO, 27 octobre, Paris. Journée des 50 ans du laser en France, histoire et futur, table ronde sur les perspectives et défis à venir.

- Journée de clôture de l'année des 50 ans du laser, 2 décembre, Hôtel de la Région, Bordeaux. Conférences scientifiques, technologiques et économiques, remise du prix Jean Jerphagnon.

\section{MANIFESTATIONS RÉGIONALES}

\section{AQUITAINE}

- "La semaine du laser en Aquitaine », 13-20 mars

- Journées nationales des procédés laser pour l'industrie, Bordeaux, 22-23 juin

- L'exposition " laser, I'odyssée aquitaine ", Bordeaux, 4 novembre - 2 décembre

\section{BRETAGNE}

- "À la lumière du laser » : nombreuses conférences (scolaires, spécialistes, tous publics), expositions et mallettes pédagogiques, festivals " Le laser en fête »

\section{ÎLE-DE-FRANCE}

- Les 50 ans du laser au CNRS

- Cycle de conférences "Découvrez le monde du laser » à l'Institut d'Optique

\section{NORD-PAS DE CALAIS}

- Cycle de conférences du CERLA, "Les 50 ans du laser », Lille

- Exposition-découverte "Laser Week » de Physifolies, Lille, 18-21 novembre

\section{PROVENCE ALPES CÔTE D’AZUR}

- Mesure de la vitesse de la lumière dans le ciel de Marseille (juin, voir article pp. 26-28) et de Nice (octobre)

- Grande Journée "Laser » à Marseille, 21 octobre

\section{RHÔNE-ALPES}

- Cycle de conférences du pôle ORA, Lyon, mars - novembre

- La semaine " 50 ans du laser » à Grenoble, 22-25 novembre

- Citons encore de nombreuses manifestations en Alsace (Strasbourg, Mulhouse), en Limousin (Limoges), en Bourgogne (Dijon)... Les scientifiques se sont mobilisés partout en France pour organiser des rencontres avec le grand public à l'occasion de la Fête de la Science, du 12 au 16 octobre. 


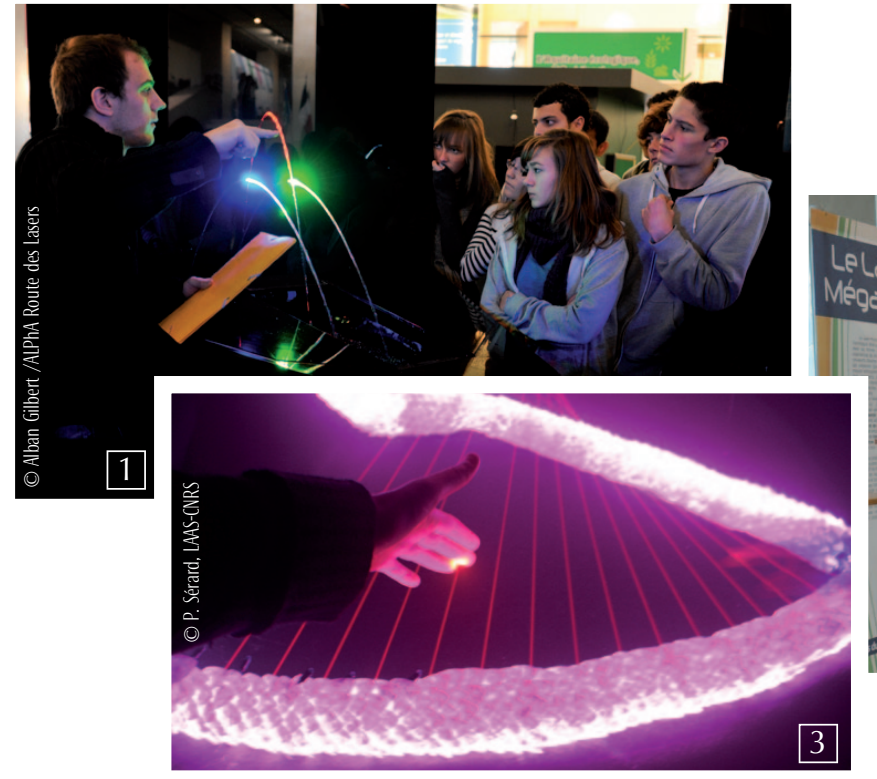

la correction de la myopie, de l'étude en profondeur de la matière à la fusion thermonucléaire..

La France a organisé, tout au long de l'année 2010, des manifestations nationales, internationales et régionales de haut prestige scientifique ou de partage de connaissances auprès des jeunes lycéens et étudiants. Ces manifestations ont fédéré, sous la houlette du Comité National, toute la communauté photonique : $\mathrm{CNOP}^{(1)}$, sociétés savantes nationales (SFO, SFP, SEE, UdPPC), $\mathrm{AFOP}^{(2)}$, pôles optiques régionaux (Optics Valley, POP Sud, ALPhA Aquitaine, Anticipa Lannion, PORA Rhône-Alpes, Rhenaphotonics Alsace, Elopsys Limoges), CNRS, Institut d'Optique, École Polytechnique, Institut de la Lumière Extrême, CEA, laboratoires de recherche, établissements d'enseignement, centres de culture scientifique, structures d'animation du territoire, plates-formes de recherche et d'innovation. Nous avons mis en route, avec l'ensemble de nos partenaires, un programme ambitieux de conférences, d'expositions fixes et itinérantes, animations, ateliers et rencontres de chercheurs,

\section{Culture scientifique}

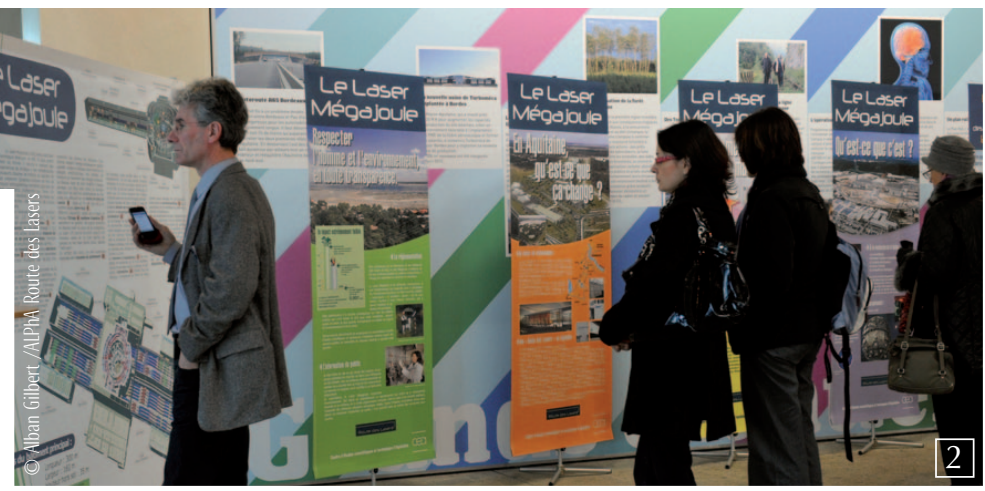

- 2 : L'exposition « laser, l'odyssée aquitaine » à Bordeaux.

: La Harpe laser à Toulouse.

visites de laboratoires et de plates-formes de R\&D, festivals, spectacles de théâtre scientifique, émissions de radio et de télévision.

Le travail du Comité a été de coordonner, valoriser, organiser et labelliser l'ensemble des manifestations organisées partout en France. Ces événements ont été découpés en trois volets : science, industrie, vie quotidienne.

Certaines animations se sont déroulées en continu durant l'année 2010. D'autres ont pris la forme d'événements mobilisateurs d'une ou plusieurs communautés, qu'elles soient scientifiques ou industrielles avec, le plus souvent, un important volet dédié aux jeunes (conférences et animations scolaires) et à la découverte des métiers associés au monde des lasers. Durant toute l'année 2010, ce sont des milliers de jeunes, chercheurs, enseignants, industriels et institutionnels, qui ont participé à cette célébration.

Costel Subran (costel.subran@optonlaser.com) Président du Comité National d'Organisation

\section{MANIFESTATIONS ARTISTIQUES}

Citons en premier la Fontaine laser géante, réalisée par le Laboratoire de physique des lasers de I'Université de Villetaneuse, en partenariat avec l'artiste Steve Veloso (voir l'article dans Reflets n²1, pp. 89-91), I'installation laser monumentale Global Rainbow réalisée par l'artiste portoricaine Yvette Mattern (festival Novela, Toulouse), la Harpe laser à Toulouse, l'animation autour de la fresque "La relativité » de Tomi Ungerer à Strasbourg, le spectacle laser à la Mairie de Paris.

\section{LIVRES, REVUES ET DOCUMENTS}

La banque d'images et le film «Les 50 ans du laser » du CNRS, le clip vidéo et la plaquette " 50 ans de laser à Paris, Ville Lumière », les livres "Le laser » (EDP Sciences) et "Le laser et ses applications » (Hermès), la plaquette "Laser » de la SFO, le numéro spécial de Reflets de la Physique/Le Bup, la série d'articles dans Photoniques, les mallettes pédagogiques réalisées en Bretagne, l'exposition itinérante « Le laser à tout faire » du CNRS, la revue Électronique Mag...

\section{SITES INTERNET}

www.50ansdulaser.fr, le site officiel qui a géré et labellisé, au nom du Comité National, toutes les manifestations qui ont eu lieu en France. Le site a compté plus de 20000 visiteurs, dont plus de 3000 étrangers. Plus de 385 sites ont créé un lien vers notre site et ont amené des visiteurs.

\section{MULTIMÉDIA}

La presse, les radios et la télévision se sont fait l'écho de ces manifestations. Les chaînes de télévision nationales ont présenté des émissions dédiées au laser. Les grands quotidiens nationaux, les journaux économiques, les magazines, les journaux régionaux ont publié des articles sur le laser.

\section{COMITÉ NATIONAL}

Président : Costel SUBRAN (CNOP, SFO)

Vice-président : André DUCASSE (CNOP, Pôle ALPhA), Responsable scientifique

Vice-présidente : Michèle LEDUC (SFP), Responsable des relations avec les grands organismes

Secrétaire Générale : Françoise MÉTIVIER (Pôle ALPHA) Trésorier : Ivan TESTART (AFOP)

Membres : Philippe AUBOURG (SFO), Pascal BESNARD (Pôle Anticipa, FOTON-ENSSAT), Marie-Pauline GACOIN (Synchrotron SOLEIL), Éric LAMBOUROUD (Optics Valley), Brigitte MACON-BOGAERT (Sciences Essonne), Paul-Éric POTTIE (SFP), Jean-Claude SIRIEYS (CNOP), Agnès ROUMIGUIERE (Pôle Anticipa Bretagne, CNOP)

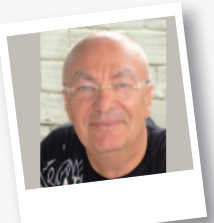

\section{Costel Subran}

Docteur ès Sciences, Costel Subran est Président Directeur général et fondateur de la société Opton Laser International, leader dans la distribution des lasers et produits photoniques en France et en Europe. Il est vice-président de la Société Française d'Optique (SFO) et du CNOP, membre du Board de "Invest in Photonics", de l'European Optical Society et de l'Optical Society of America ; enfin, il est membre fondateur du Comité de rédaction de la revue Photoniques et administrateur de EDP Sciences. 